\title{
Ophiopogonin D attenuates PM2.5-induced inflammation via suppressing the AMPK/NF-кB pathway in mouse pulmonary epithelial cells
}

\author{
YING WANG ${ }^{1,2}$, DAN LI $^{1}$, LEI SONG ${ }^{1}$ and HUI DING ${ }^{1}$ \\ ${ }^{1}$ Department of Respiratory Medicine, The First Hospital of Jilin University, Changchun, Jilin 130021; \\ ${ }^{2}$ Department of Pulmonary and Critical Care Medicine, Peking University Third Hospital, Beijing 100191, P.R. China
}

Received October 16, 2019; Accepted July 24, 2020

DOI: $10.3892 /$ etm.2020.9268

\begin{abstract}
Exposure to fine particulate matter, such as particulate matter of $\leq 2.5 \mu \mathrm{m}$ in diameter (PM2.5), causes pulmonary inflammation and injury to other organs. It has been reported that Ophiopogonin D (OP-D) has anti-inflammatory activity. The aim of the present study was to investigate this anti-inflammatory activity of OP-D on PM2.5-induced acute airway inflammation and its underlying mechanisms. The viability of PM2.5-treated mouse lung epithelial (MLE-12) cells with or without OP-D treatment was determined using a Cell Counting Kit- 8 assay. The corresponding levels of IL-1 $\beta$, IL-6, IL- 8 and TNF- $\alpha$ were examined via ELISA. Subcellular localization of $\mathrm{NF}-\kappa \mathrm{Bp} 65$ was detected using immunofluorescence staining. The expression levels of AMP-activated protein kinase (AMPK), phosphorylated (p)-AMPK,

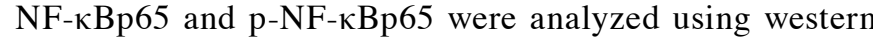
blotting. The selective AMPK inhibitor Compound C (CC) was utilized to investigate the involvement of AMPK in the protection against PM2.5-induced cell inflammation by OP-D treatment. The results demonstrated that OP-D significantly ameliorated the PM2.5-stimulated release of proinflammatory cytokines (TNF- $\alpha$, IL-1 $\beta$, IL- 6 and IL-8) and inhibited the translocation of NF- $\kappa$ Bp65 from the cytoplasm to the nucleus in MLE-12 cells. Moreover, OP-D significantly prevented the PM2.5-triggered phosphorylation of NF- $\mathrm{Bp} 65$ and upregulated AMPK activity. The anti-inflammatory activity of OP-D could also be attenuated by the AMPK-specific inhibitor $\mathrm{CC}$. The present results suggested that the anti-inflammatory activity of OP-D was mediated via AMPK activation and $\mathrm{NF}-\kappa \mathrm{B}$ signaling pathway downregulation, which ameliorated the expression of proinflammatory cytokines. Therefore, OP-D
\end{abstract}

Correspondence to: Dr Hui Ding, Department of Respiratory Medicine, The First Hospital of Jilin University, 71 Xinmin Street, Changchun, Jilin 130021, P.R. China

E-mail:dinghui08@sina.com

Key words: AMP-activated protein kinase, NF- $\mathrm{BB}$, inflammation, Ophiopogonin $\mathrm{D}$, particulate matter of $\leq 2.5 \mu \mathrm{m}$ in diameter could be a candidate drug to treat PM2.5-induced airway inflammation.

\section{Introduction}

Pollution with particulate matter of $\leq 2.5 \mu \mathrm{m}$ in diameter (PM2.5) is known to have significant deleterious effects on human health (1-3). The respiratory system exchanges gases with the ambient gas environment directly; therefore, PM2.5-containing air can cause airway lesions (4). The increase of inflammatory cytokines has been revealed to be positively correlated with the exposure dose and duration (5), and even a low dose of PM2.5 can cause inflammation and pulmonary injury (6). Moreover, PM2.5 has a cumulative effect on the respiratory system. For instance, the longer the exposure time, the stronger the adverse influence of PM2.5 to the respiratory system (7). Previous studies have reported that PM2.5 can activate the NF- $\kappa$ B pathway, as well as upregulate the transcription and secretion of proinflammatory cytokines, including IL-1 $\beta$, TNF- $\alpha$, IL-6 and IL-8, ultimately inducing widespread pulmonary inflammatory lesions $(8,9)$. In addition, it has been observed that upregulation of AMP-activated protein kinase (AMPK) suppresses the inflammatory response via inhibition of inflammatory cytokines and NF- $\mathrm{KB}(10,11)$. Therefore, downregulation of the $\mathrm{NF}-\kappa \mathrm{B}$ signaling pathway or activation of AMPK may be a valid method for controlling PM2.5-induced respiratory inflammation and preventing or limiting lung injury.

Ophiopogon japonicus (O. japonicus; commonly known as Maidong in China) was first recorded in Shen Nong's Materia Medica written during the Han dynasty and has been widely applied in traditional Chinese medicine (12). Currently, O. japonicus is often used in compound prescriptions as the main medicinal ingredient, such as in YiQiFuMai injection, Sheng Mai Yin and Xuanmai granule (13). According to the Chinese Pharmacopoeia, O. japonicus has been applied for $1,000 \mathrm{~s}$ of years for the treatment of inflammatory diseases, such as pharyngitis, bronchitis, pneumonia and cough (14). Ophiopogonin D (OP-D) is a vital bioactive steroidal glycoside extracted from the root of $O$. japonicas (15). Accumulating evidence has indicated that OP-D possesses a broad range of pharmacological properties, including anti-inflammatory, 
antioxidative and antitussive effects, as well as inhibition of venous thrombosis (16-18). However, it remains unknown whether OP-D is able to protect alveolar epithelial cells from PM2.5-induced toxicity via its anti-inflammatory effects. Therefore, it was hypothesized that OP-D may be potentially useful for preventing PM2.5-induced pulmonary inflammation.

The aim of the present study was to investigate the protective effects of OP-D on PM2.5-induced pulmonary inflammation. In addition, the molecular mechanisms of the anti-inflammatory effects of OP-D were evaluated.

\section{Materials and methods}

Materials. OP-D, extracted from O. japonicus, was obtained from Beijing Biotop Biotechnology Development (cat. no. 41753-55-3), dissolved in DMSO (Sigma-Aldrich; Merck KGaA), and diluted with basal DMEM (Beijing Transgen Biotech) (19). The final concentration of DMSO in the culture medium was $0.1 \%(\mathrm{v} / \mathrm{v})$. The use of $0.1 \%$ DMSO alone in DMEM was used as a negative control in the corresponding experiments.

The AMPK-specific inhibitor Compound C (CC) was purchased from Selleck Chemicals (cat. no. S7306). Streptomycin, penicillin, FBS and trypsin were obtained from Gibco (Thermo Fisher Scientific, Inc.). A BCA Protein assay kit (cat. no. 23250) and an Enhanced Chemiluminescence western blotting Detection reagents kit were obtained from Thermo Fisher Scientific. The CelLytic ${ }^{\mathrm{TM}}$ NuCLEAR $^{\mathrm{TM}}$ Extraction kit was purchased from Sigma-Aldrich; Merck KGaA (cat. no. NXTRACT-1KT). ELISA kits for IL-1 $\beta$ (cat. no. BPE20083), IL-6 (cat. no. BPE20012), IL-8 (cat. no. BPE20459) and TNF- $\alpha$ (cat. no. BPE20220) were obtained from Shanghai Lengton Bioscience Co., Ltd. The Cell Counting Kit-8 (CCK-8) was from Beijing Transgen Biotech (cat. no. FC101-04).

Antibodies against AMPK (1:1,000; cat. no. A1229), phosphorylated (p)-AMPK $\beta 1$-S108 (1:800; cat. no. AP0597), p-NF-кBp65 (1:1,000; cat. no. AP0417), NF-кBp65 (1:1,000; cat. no. A14754), lamin B (1:2,000; cat. no. A1910) and GAPDH (1:2,000; cat. no. AC033), as well as HRP goat anti-rabbit antibody (1:5,000; cat. no. AS014), HRP goat anti-mouse antibody (1:5,000; cat. no. AS003) and FITC goat anti-rabbit antibody (1:80; cat. no. AS011), were obtained from ABclonal Biotech. All other reagents were obtained from Sigma-Aldrich; Merck KGaA.

Cell culture. Mouse lung epithelial cells (MLE-12; The Cell Bank of Type Culture Collection of the Chinese Academy of Sciences) were cultured in complete DMEM containing $10 \%$ (v/v) FBS, $100 \mathrm{U} / \mathrm{ml}$ penicillin, $100 \mathrm{~g} / \mathrm{ml}$ streptomycin and $50 \mathrm{~g} / \mathrm{ml}$ amphotericin $\mathrm{B}$ at $37^{\circ} \mathrm{C}$ in an incubator with $5 \%$ atmospheric $\mathrm{CO}_{2}$. The media was replaced every 2-3 days, and the cells were subcultured weekly $\left(1,500-3,000\right.$ cells $\left./ \mathrm{cm}^{2}\right)$.

PM2.5 collection and preparation. PM2.5 was collected between November 2016 and March 2017 in an urban area of Changchun, Jilin. The collection and preparation procedures of PM2.5 were the same as those reported previously (20). Briefly, concentrated PM2.5 was gathered using a multistage particle counter $(100 \mathrm{l} / \mathrm{min}$; total suspended
particulate/PM10/PM5/PM2.5; Laoshan Electronic Instrument Co.,Ltd.). Daily PM2.5 samples were collected on Teflon filters (90 mm; Whatman plc; Cytiva). The PM-loaded filters were put into a 50-ml centrifuge tube, followed by probe-sonication $\left(700 \mathrm{~W} ; 40 \mathrm{kHz} ; 25^{\circ} \mathrm{C}\right.$ ) for $1 \mathrm{~h}$ in $40 \mathrm{ml}$ Milli-Q water. The suspension was freeze-dried in a vacuum for $12 \mathrm{~h}$ to obtain PM2.5 powder, which was then weighed and stored at $-80^{\circ} \mathrm{C}$. Before the experiments, the PM2.5 powder was dissolved in PBS and sonicated $\left(700 \mathrm{~W} ; 40 \mathrm{kHz} ; 25^{\circ} \mathrm{C}\right)$ for $30 \mathrm{~min}$ to avoid aggregation of particles.

Cell viability assay. Cell viability was assessed using a CCK-8 assay, according to the manufacturer's protocol. A total of $4 \times 10^{3}$ MLE-12 cells per well in $100 \mu 1$ media were cultivated in 96-well plates and grown at $37^{\circ} \mathrm{C}$ for $24 \mathrm{~h}$. The cells were treated with OP-D $(0-320 \mu \mathrm{M})$ for $1 \mathrm{~h}$ at $37^{\circ} \mathrm{C}$ and then reacted with or without $15 \mu \mathrm{g} / \mathrm{cm}^{2} \mathrm{PM} 2.5$ at $37^{\circ} \mathrm{C}$ in an incubator for $24 \mathrm{~h}$. CCK-8 solution (10 $\mu \mathrm{l})$ was added into each well, and the cells were incubated for another $4 \mathrm{~h}$ at $37^{\circ} \mathrm{C}$. Finally, the absorbance of each well was measured at a wavelength of $450 \mathrm{~nm}$ using a microplate reader. Each sample assay was repeated three times.

Immunofluorescence staining. MLE-12 cells ( $1 \times 10^{5}$ cells/well) on a glass coverslip cultured in 24-well plates were treated with $15 \mu \mathrm{g} / \mathrm{cm}^{2}$ PM2.5 and $80 \mu \mathrm{M}$ OP-D, cells were then fixed with $4 \%$ paraformaldehyde, blocked with $5 \%$ BSA (cat. no. A1933-25G; Sigma-Aldrich; Merck KGaA) for 30 min at room temperature (RT) and incubated with anti-NF-кBp65 (1:200) overnight at $4^{\circ} \mathrm{C}$. After washing with PBS, the coverslip was treated with secondary antibody $(1: 80)$ for $1 \mathrm{~h}$ at RT. Following washing with PBS, the nuclei were stained with DAPI (Invitrogen; Thermo Fisher Scientific, Inc.) for $20 \mathrm{~min}$ at RT. The stained cells were then observed under an FV-1,000 fluorescence microscope (Olympus Corporation) under x400 magnification. Semi-quantitative analysis was performed using ImageJ 1.51t software (National Institutes of Health).

ELISA. Based on the experimental groups, the MLE-12 cells $\left(7 \times 10^{5}\right.$ cells/well) were cultivated in six-well plates, pretreated with OP-D at $10,20,40$ or $80 \mu \mathrm{M}$ with $10 \mu \mathrm{M} \mathrm{CC}$ for $1 \mathrm{~h}$, and then stimulated with $15 \mu \mathrm{g} / \mathrm{cm}^{2} \mathrm{PM} 2.5$ for $24 \mathrm{~h}$ at $37^{\circ} \mathrm{C}$. The PM2.5 group was stimulated with the corresponding dose of PM2.5. The OP-D 80 group was only treated with $80 \mu \mathrm{M}$ OP-D. After incubation, the cultured media samples were collected to determine the levels of IL-1 $\beta$, IL- 6 , IL-8 and TNF- $\alpha$, using Mouse Quantikine ELISA kits, according to the manufacturer's instructions. The cells were harvested for western blot analysis.

Western blotting. Cell lysates were extracted via homogenization with RIPA buffer (Beyotime Institute of Biotechnology) containing phenylmethanesulfonyl fluoride, protease inhibitors and phosphatase inhibitors. The nuclear fraction was isolated using a Nuclear and Cytoplasmic extraction kit (cat. no. NXTRACT-1KT; Sigma-Aldrich; Merck KGaA). The concentration of each supernatant was determined using a BCA protein assay kit. Aliquoted proteins $(60 \mu \mathrm{g} /$ well) were loaded onto a $10 \%$ SDS-PAGE, separated by electrophoresis and then transferred onto a PVDF membrane. After blocking 
A

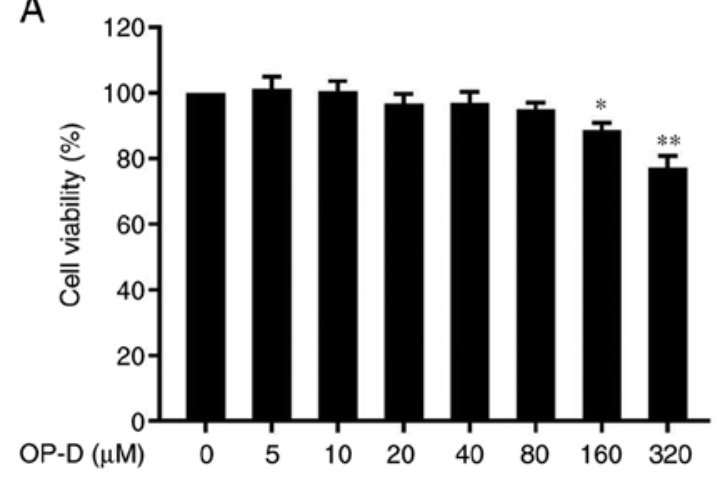

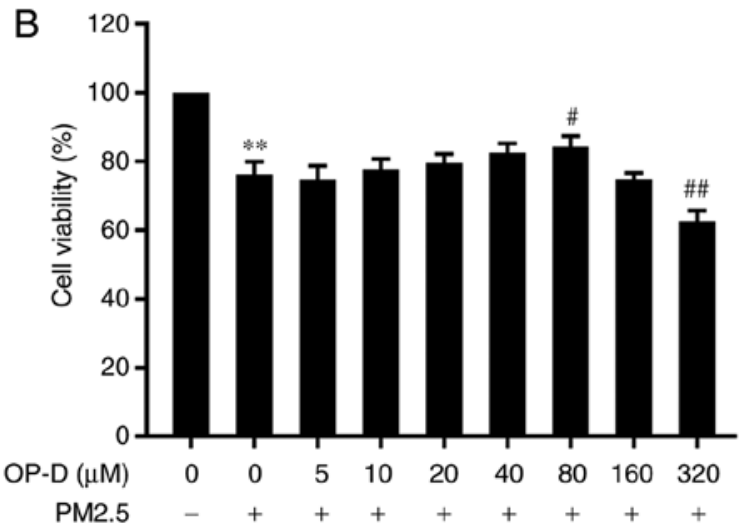

Figure 1. Effect of OP-D on the cellular viability of PM2.5-treated MLE-12 cells. (A) The cellular cytotoxicity of OP-D was evaluated in MLE-12 cells at the indicated concentrations. (B) Cells were pretreated with OP-D $(0-320 \mu \mathrm{M})$ for $1 \mathrm{~h}$ and then stimulated with PM2.5 $\left(15 \mu \mathrm{g} / \mathrm{cm}^{2}\right)$ for $24 \mathrm{~h}$. The cellular viability was detected. Data are presented as the mean $\pm \mathrm{SD}$ for three independent assays. ${ }^{*} \mathrm{P}<0.05$ and ${ }^{* *} \mathrm{P}<0.01$ vs. OP-D control group $\left(0 \mu \mathrm{M}\right.$ OP-D); ${ }^{*} \mathrm{P}<0.05$ and ${ }^{\# \#} \mathrm{P}<0.01$ vs. PM2.5-treated group $\left(0 \mu \mathrm{M}\right.$ OP-D with $\left.15 \mu \mathrm{g} / \mathrm{cm}^{2} \mathrm{PM} 2.5\right)$. OP-D, Ophiopogonin D; PM2.5, particulate matter of $\leq 2.5 \mu \mathrm{m}$ in diameter.

with 5\% skimmed milk in Tris-buffered saline-Tween-20 (TBS-T, $0.1 \%$ Tween-20) for $1 \mathrm{~h}$ at RT, the membrane was treated with the corresponding primary antibody (AMPK, p-AMPK, NF- $\kappa$ Bp 65, p-NF- $\kappa$ Bp65, GAPDH or lamin B) at $4^{\circ} \mathrm{C}$ overnight, washed with TBST buffer three times and then treated with horseradish peroxidase-conjugated secondary antibody for $1 \mathrm{~h}$ at RT. After washing with TBST three times, the membranes were visualized with enhanced chemiluminescence reagents. GAPDH and lamin B were employed as cytosol and nuclear protein loading controls, respectively. Semi-quantitative analysis was performed using ImageJ $1.51 \mathrm{t}$ software.

Statistical analysis. Data are presented as the mean \pm SD. Statistical analysis was performed using SPSS 20.0 software (IBM Corp.). Comparison of cell survival rate between the groups were performed using two-way ANOVA, while other experimental groups were compared using one-way ANOVA followed by Bonferroni's post hoc test. Experiments were repeated three times. $\mathrm{P}<0.05$ was considered to indicate a statistically significant difference.

\section{Results}

OP-D prevents the PM2.5-induced decrease of MLE-12 cell viability. Cellular viability was assayed in the OP-D $(0,5$, 10, 20, 40, 80, 160 and $320 \mu \mathrm{M}$ )-treated MLE-12 cells. No cellular cytotoxicity was observed at OP-D concentrations of $0-80 \mu \mathrm{M}$ for $24 \mathrm{~h}$ in the presence or absence of PM2.5 (Fig. 1). However, higher concentrations (160 and $320 \mu \mathrm{M}$ ) of OP-D caused significant cytotoxicity, thus reducing the cellular viability (Fig. 1A). PM2.5 significantly decreased the MLE-12 cellular viability, compared with the untreated cells (Fig. 1B). Moreover, pretreatment with $80 \mu \mathrm{M}$ OP-D for $1 \mathrm{~h}$ significantly attenuated the PM2.5-induced decrease of cellular viability (Fig. 1B). Lower concentrations $(5-80 \mu \mathrm{M})$ of OP-D did not affect cellular viability, however, $320 \mu \mathrm{M}$ OP-D significantly further inhibited cellular viability after PM2.5 treatment (Fig. 1B). Therefore, concentrations of 10 , 20, 40 and $80 \mu \mathrm{M}$ OP-D were selected for the subsequent experiments.
OP-D attenuates the PM2.5-induced levels of TNF- $\alpha, I L-6$, $I L-8$ and $I L-1 \beta$ in MLE-12 cells. The anti-inflammatory activity of OP-D was investigated in PM2.5-treated MLE-12 cells via ELISA to examine levels of IL-1 $\beta$, IL-6, IL-8 and TNF- $\alpha$ in the culture supernatant. PM2.5 treatment significantly induced the expression of IL-1 $\beta$ (Fig. 2A), IL-6 (Fig. 2B), IL-8 (Fig. 2C) and TNF- $\alpha$ (Fig. 2D) in the culture medium of MLE-12 cells, compared with the negative controls. Cells treated with OP-D alone did not demonstrate any effect on the levels of proinflammatory cytokines. However, pretreatment with OP-D at 20-80 $\mu \mathrm{M}$ significantly reduced the levels of the PM2.5-triggered cytokines in a dose-dependent manner, compared with those of the control PM2.5-treated cells (Fig. 2).

$O P-D$ inhibits the $N F-\kappa B$ inflammatory signaling induced by PM2.5 in MLE-12 cells. OP-D has been reported to reduce inflammation via activation of the NF- $\kappa B$ signaling pathway (19), which can be suppressed by AMPK. To further examine the anti-inflammatory activity of OP-D, the effects of OP-D were detected on both NF- $\kappa \mathrm{B}$ subcellular translocation and the protein phosphorylation of AMPK and NF- $\kappa$ Bp65 in MLE-12 cells using immunofluorescence and western blot analysis.

PM2.5 treatment significantly induced $N F-\kappa B$ translocation from the cytoplasm to the nucleus, compared with the negative control in which most $\mathrm{NF}-\kappa \mathrm{B}$ expression still remained in the cytoplasm, according to immunofluorescence staining (Fig. 3B). In contrast, the PM2.5-induced nuclear translocation of NF- $\kappa \mathrm{Bp} 65$ was partly suppressed by pretreatment with OP-D (Fig. 3). Taken together, these findings suggest the potential anti-inflammatory effect of OP-D in PM2.5-induced cellular inflammation.

OP-D phosphorylates AMPK in PM2.5-treated MLE-12 cells. Next, the activation of AMPK in MLE-12 cells was investigated via western blot analysis. Exposure to PM2.5 did not significantly alter the phosphorylation of AMPK; however, 10-80 $\mu \mathrm{M}$ OP-D significantly increased the phosphorylation of AMPK in a dose-dependent manner (Fig. 4A). Moreover, there were no significant changes in the OP-D alone group. 

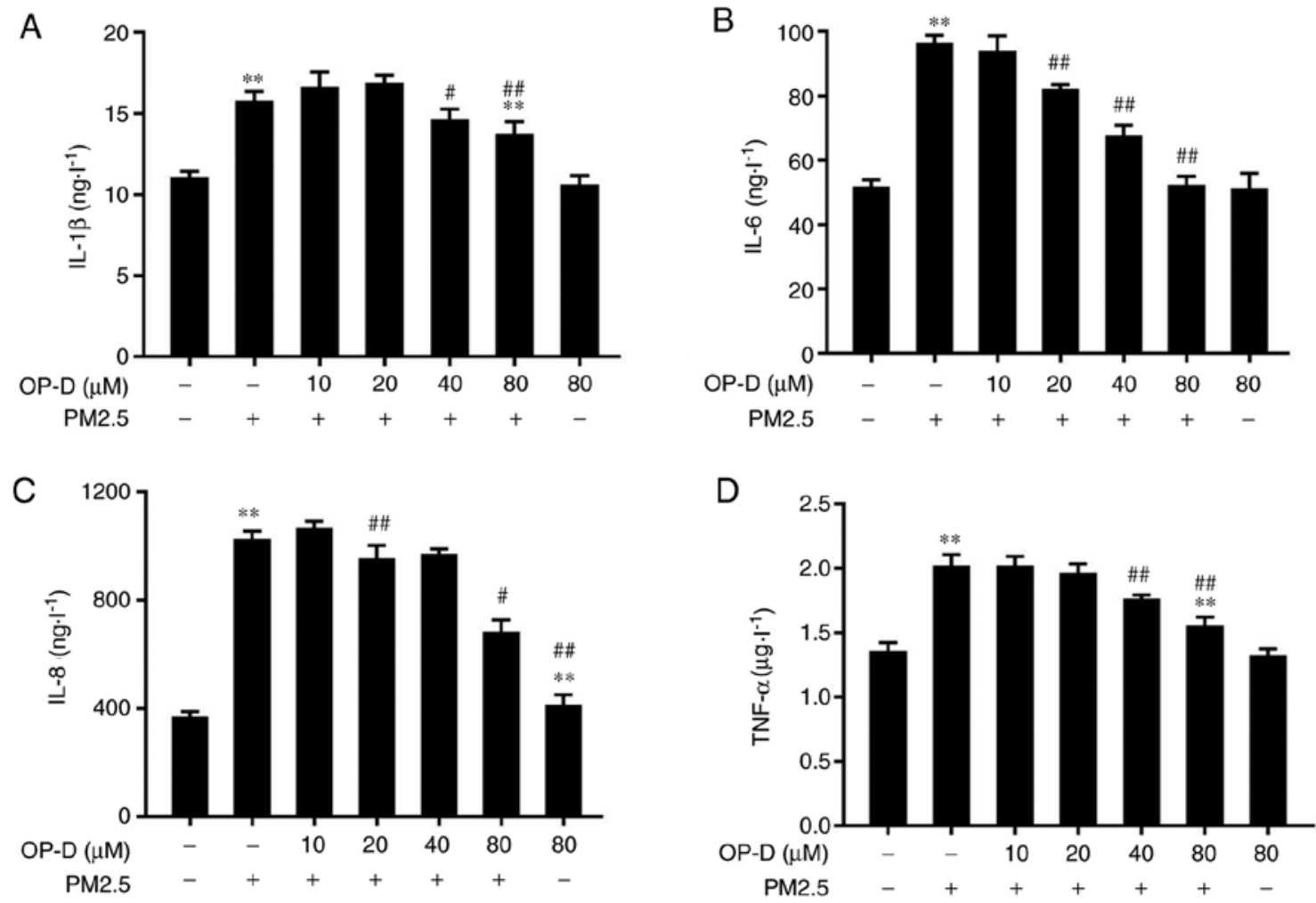

Figure 2. OP-D inhibits PM2.5-induced proinflammatory cytokine expression of IL-1 $\beta$, IL-6, IL-8 and TNF- $\alpha$ in MLE-12 cells. MLE-12 cells were pretreated with the indicated concentrations of OP-D for $1 \mathrm{~h}$ and then stimulated with PM2.5 (15 $\left.\mu \mathrm{g} / \mathrm{cm}^{2}\right)$ for another $24 \mathrm{~h}$. The levels of (A) IL-1 $\beta$, (B) IL-6, (C) IL-8 and (D) TNF- $\alpha$ in the culture medium were determined using ELISA. Data are presented as the mean $\pm \mathrm{SD}(\mathrm{n}=5) .{ }^{* *} \mathrm{P}<0.01$ vs. controls; ${ }^{\#} \mathrm{P}<0.05$ and ${ }^{\# \#} \mathrm{P}<0.01$ vs. PM2.5-treated cells. OP-D, Ophiopogonin D; PM2.5, particulate matter of $\leq 2.5 \mu \mathrm{m}$ in diameter.
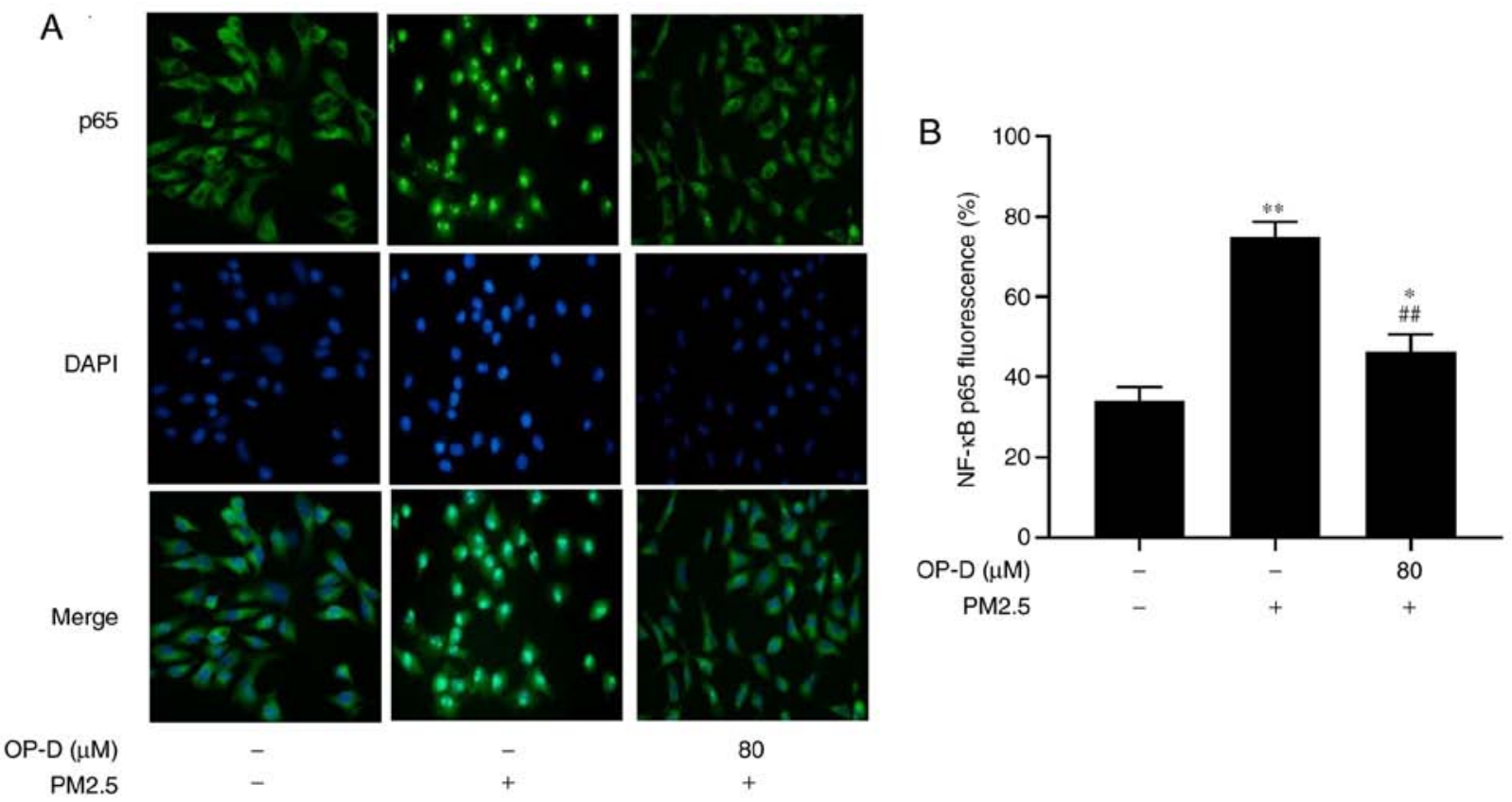

Figure 3. Immunofluorescence staining of NF- $\mathrm{\kappa Bp} 65$ in MLE-12 cells. MLE-12 cells were pretreated with $80 \mu \mathrm{M}$ OP-D for $1 \mathrm{~h}$ and then stimulated with $15 \mu \mathrm{g} / \mathrm{cm}^{2}$ PM2.5 for 24 h. (A) NF-kBp65 (green) was visualized via immunofluorescence staining. Nuclei were stained by DAPI (blue). Magnification, x400. (B) The overall fluorescence was semi-quantified. Data are presented as the mean $\pm \mathrm{SD}(\mathrm{n}=3)$. ${ }^{*} \mathrm{P}<0.05$ and ${ }^{* * *} \mathrm{P}<0.01$ vs. controls; ${ }^{* \#} \mathrm{P}<0.01$ vs. $\mathrm{PM} 2.5$-treated cells. OP-D, Ophiopogonin D; PM2.5, particulate matter of $\leq 2.5 \mu \mathrm{m}$ in diameter.

OP-D inhibits PM2.5-triggered NF- $\kappa B$ activation. PM2.5 exposure significantly phosphorylated $\mathrm{NF}-\kappa \mathrm{B}$, and pretreatment with OP-D (20-80 $\mu \mathrm{M})$ significantly attenuated this activation in a dose-dependent manner (Fig. 4A) in MLE-12 cells. In addition, similar to the immunofluorescence staining results (Fig. 3), PM2.5 treatment significantly decreased NF- $\mathrm{KB}$ expression in 
A
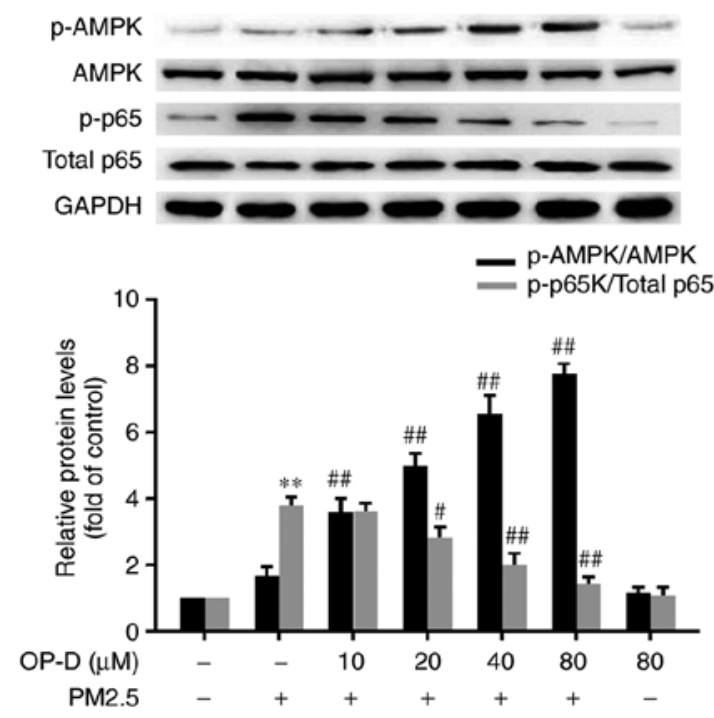

B
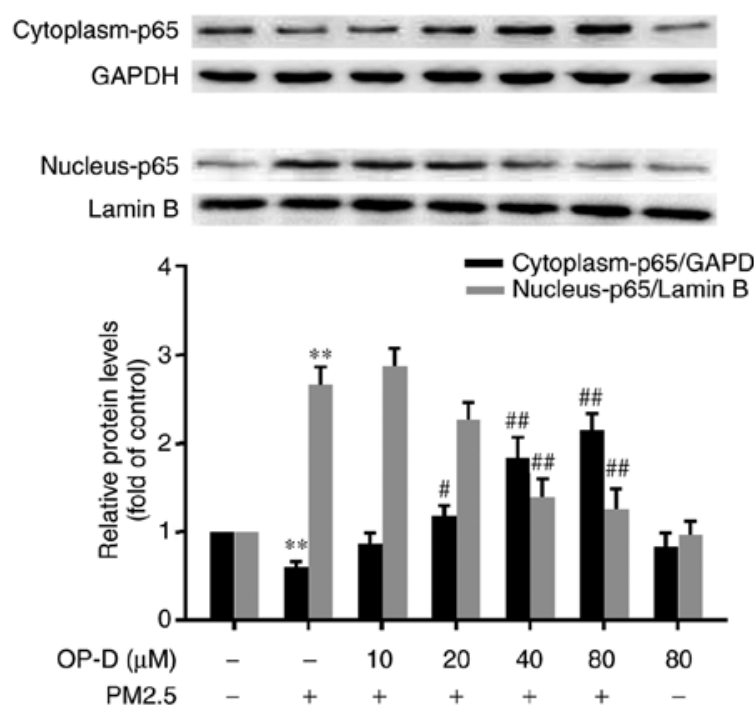

Figure 4. OP-D phosphorylated AMPK and inhibited PM2.5-induced NF- $\kappa$ B activation in MLE-12 cells. Representative western blotting images demonstrating (A) the activation state of p-AMPK and p-p65 in $15 \mu \mathrm{g} / \mathrm{cm}^{2} \mathrm{PM} 2.5$-treated $(24 \mathrm{~h}) \mathrm{MLE}-12$ cells with or without pretreatment of OP-D (1 h) at different concentrations. (B) Expression of p-p65 was detected in the isolated cytoplasm and nuclei. Total AMPK, NF- $\kappa$ B, GAPDH and lamin B were used as the corresponding protein loading references. The relative phosphorylation levels of AMPK (to total AMPK), NF- $\kappa \mathrm{B}$ (to total NF- $\kappa \mathrm{B}$ ), cytoplasmic NF- $\kappa \mathrm{B}$ (to GAPDH) and nuclear $\mathrm{NF}-\kappa \mathrm{B}$ (to lamin $\mathrm{B}$ ) were analyzed. The relative phosphorylation data are presented as the mean $\pm \mathrm{SD}$ ( $\mathrm{n}=3$ in each group). ${ }^{* *} \mathrm{P}<0.01$ vs. controls; ${ }^{\#} \mathrm{P}<0.05$ and ${ }^{\# \#} \mathrm{P}<0.01$ vs. PM2.5-treated cells. AMPK, AMP-activated protein kinase; OP-D, Ophiopogonin D; PM2.5, particulate matter of $\leq 2.5 \mu$ m in diameter; p-, phosphorylated.

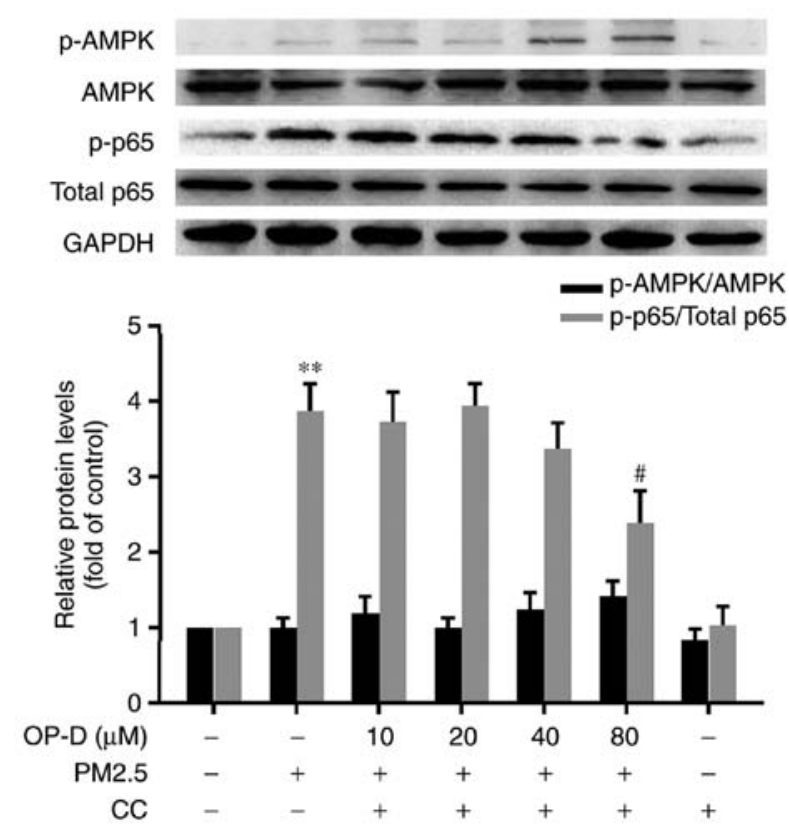

Figure 5. CC blocks the OP-D-mediated inhibition of NF- $\kappa \mathrm{B}$ activation in PM2.5-exposed MLE-12 cells. MLE-12 cells were pretreated with different concentrations of OP-D $(10,20,40$ or $80 \mu \mathrm{M})$ and $10 \mu \mathrm{M} \mathrm{CC}$ for $1 \mathrm{~h}$, and then they were exposed to $15 \mu \mathrm{g} / \mathrm{cm}^{2} \mathrm{PM} 2.5$ for $24 \mathrm{~h}$. The expression levels of $\mathrm{NF}-\kappa \mathrm{Bp} 65, \mathrm{p}-\mathrm{NF}-\kappa \mathrm{Bp} 65, \mathrm{AMPK}$ and $\mathrm{p}-\mathrm{AMPK}$ were assessed using western blotting and were semi-quantified. Data are presented as the mean \pm SD ( $\mathrm{n}=3$ in each group). ${ }^{* *} \mathrm{P}<0.01$ vs. controls; ${ }^{*} \mathrm{P}<0.05$ vs. PM2.5-treated cells. AMPK, AMP-activated protein kinase; CC, Compound C; OP-D, Ophiopogonin D; PM2.5, particulate matter of $\leq 2.5 \mu \mathrm{m}$ in diameter; p-, phosphorylated.

the cytoplasm, while NF- $\kappa \mathrm{B}$ expression in the nuclei was significantly increased. These alterations were significantly reversed by pretreatment with OP-D in a dose-dependent manner (Fig. 4B).
$C C$ inhibits the $O P-D$-induced dephosphorylation of $N F-\kappa B$ in MLE-12 cells. To elucidate whether the anti-inflammatory effect of OP-D is mediated via AMPK, all the OP-D-treated cells were incubated with $10 \mu \mathrm{M}$ CC simultaneously. The CC-treated cells did not demonstrate any significant activation of AMPK (Fig. 5). In the PM2.5-exposed MLE-12 cells cotreated with OP-D and CC, the 20-40 $\mu \mathrm{M}$ OP-D-mediated decrease of $\mathrm{NF}-\kappa \mathrm{B}$ was significantly blocked; however, $\mathrm{CC}$ did not inhibit this effect in the $80 \mu \mathrm{M}$ OP-D-treated cells compared with the negative control cells (Fig. 5). These results indicated that AMPK mediated $\mathrm{NF}-\kappa \mathrm{B}$ activation during PM2.5-induced inflammation.

$C C$ upregulates the levels of TNF- $\alpha, I L-6, I L-8$ and $I L-1 \beta$ inhibited by OP-D in MLE-12 cells. The aforementioned results suggested that the anti-inflammatory activity of OP-D is AMPK dependent. In order to verify this finding, the downregulation of IL-1 $\beta$ (Fig. 6A), IL-6 (Fig. 6B), IL-8 (Fig. 6C) and TNF- $\alpha$ (Fig. 6D) by OP-D was measured in the presence or absence of CC. CC alone did not demonstrate any notable effect on the levels of the proinflammatory cytokines. Pretreatment with $10 \mu \mathrm{M}$ CC significantly reversed the downregulation of IL-1 $\beta$, IL- 6 , IL- 8 and TNF- $\alpha$ induced by OP-D $(80 \mu \mathrm{M})$, compared with the group without $\mathrm{CC}$ treatment. Furthermore, the inhibition by OP-D did not completely reverse to the control levels of TNF- $\alpha$, IL- 8 and IL-1 $\beta$, compared with the PM2.5-treated group. These results indicated that downregulation of the inflammatory factors by OP-D was partly via the AMPK pathway, which is consistent with the western blotting results. Thus, the present results suggested that OP-D attenuated the PM2.5-induced cell inflammation via activation of AMPK and suppression of the $\mathrm{NF}-\kappa \mathrm{B}$ signaling pathway. 
A
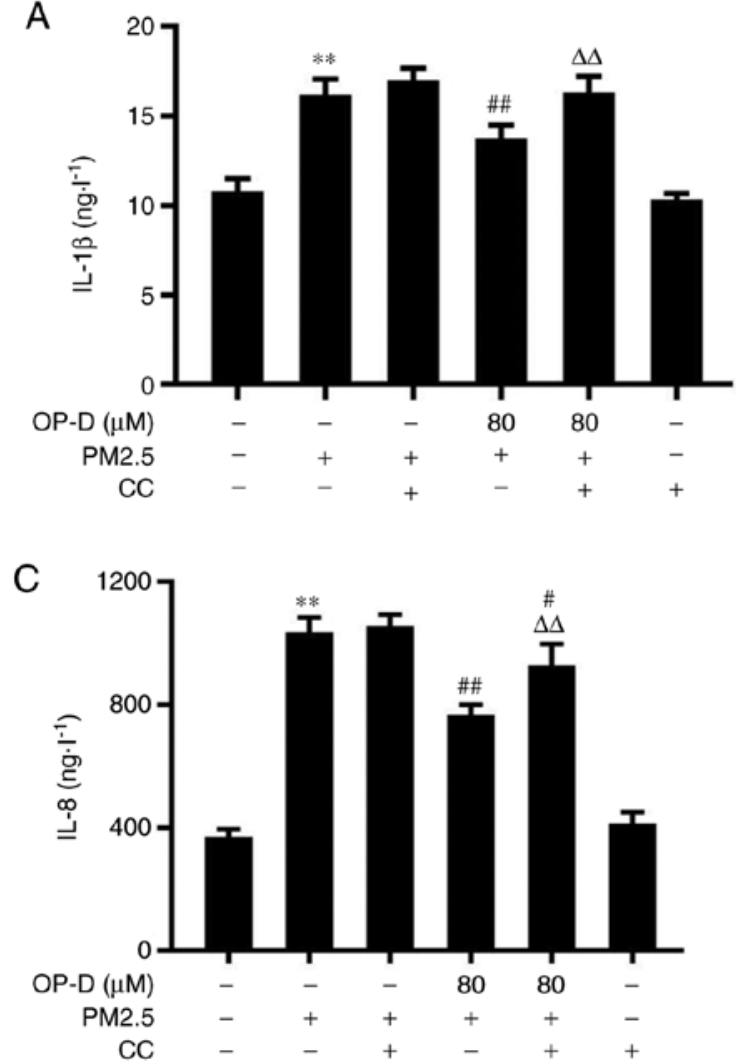

B

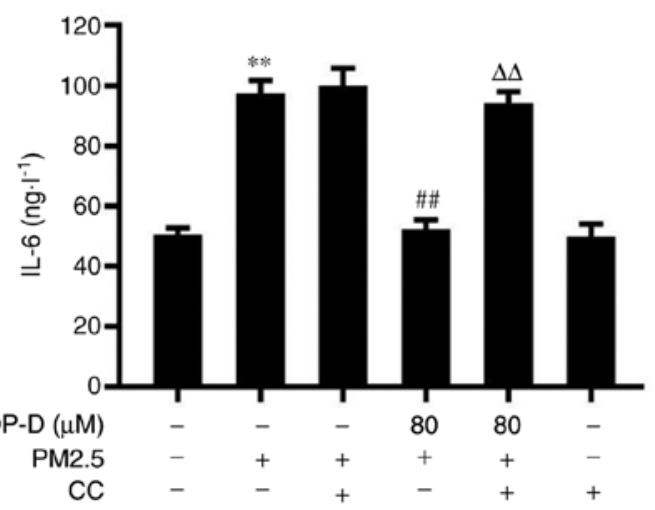

D

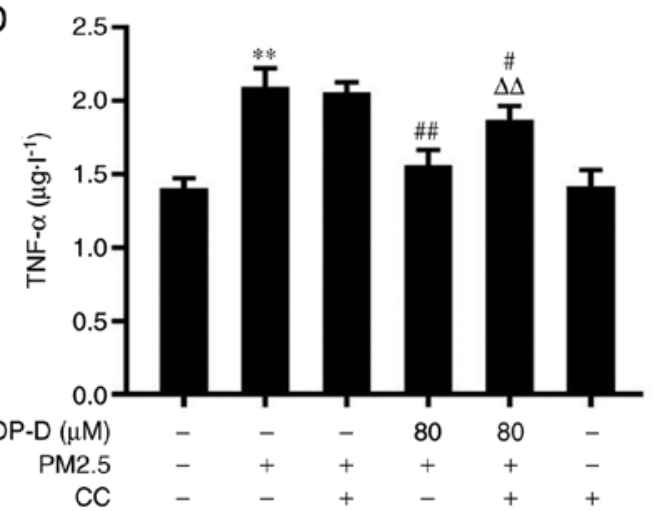

Figure 6. CC reverses the OP-D-mediated inhibition of proinflammatory cytokine expression in MLE-12 cells. MLE-12 cells were pretreated with different concentrations of OP-D $(10,20,40$ or $80 \mu \mathrm{M})$ and $10 \mu \mathrm{M} \mathrm{CC}$ for $1 \mathrm{~h}$, and then they were exposed to $15 \mu \mathrm{g} / \mathrm{cm}^{2}$ PM2.5 for $24 \mathrm{~h}$. The levels of (A) IL-1 13 , (B) IL-6, (C) IL-8 and (D) TNF- $\alpha$ in the culture medium were determined via ELISA. Data are presented as the mean $\pm \mathrm{SD}(\mathrm{n}=5)$. ${ }^{* *} \mathrm{P}<0.01 \mathrm{vs}$. controls; ${ }^{*} \mathrm{P}<0.05$ and ${ }^{\# \#} \mathrm{P}<0.01$ vs. PM2.5-treated cells; ${ }^{\Delta \Delta} \mathrm{P}<0.01$ vs. PM $2.5+80 \mu \mathrm{M}$ OP-D group. CC, Compound C; OP-D, Ophiopogonin D; PM2.5, particulate matter of $\leq 2.5 \mu \mathrm{m}$ in diameter.

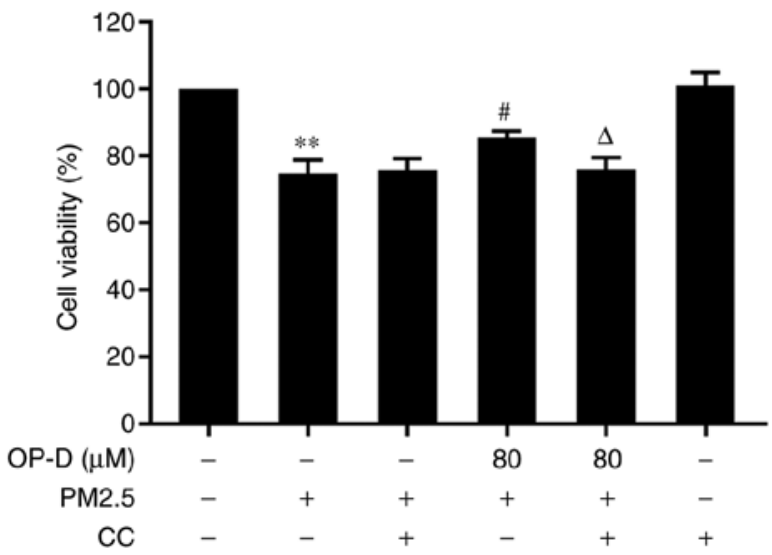

Figure 7. Effect of CC on the cellular viability of PM2.5-treated MLE-12 cells. MLE-12 cells were pretreated with different concentrations of OP-D $(10,20,40$ and $80 \mu \mathrm{M})$ and $10 \mu \mathrm{M} \mathrm{CC}$ for $1 \mathrm{~h}$, and then exposed to $15 \mu \mathrm{g} / \mathrm{cm}^{2}$ PM2.5 for $24 \mathrm{~h}$. Data are presented as the mean \pm SD for three independent assays. ${ }^{* *} \mathrm{P}<0.01$ vs. controls; ${ }^{~} \mathrm{P}<0.05$ vs. $\mathrm{PM} 2.5$-treated cells; ${ }^{\Delta} \mathrm{P}<0.05$, vs. $\mathrm{PM} 2.5+80 \mu \mathrm{M}$ OP-D treated group. CC, Compound C; OP-D, Ophiopogonin $\mathrm{D}$; PM2.5, particulate matter of $\leq 2.5 \mu \mathrm{m}$ in diameter.

$C C$ reduces the protective effect of $O P-D$ in MLE cells. $\mathrm{CC}$ was used to block the activation of AMPK in order to observe whether the AMPK pathway is essential to protect cellular viability. The present results demonstrated that blocking the AMPK pathway with CC significantly reduced the protective effect of OP-D on the PM2.5-induced cells (Fig. 7; P<0.05). These results indicated that OP-D had a protective effect on MLE cells via activation of the AMPK signaling pathway.

\section{Discussion}

To the best of our knowledge, the present study was the first to demonstrate that OP-D has an anti-inflammatory effect on PM2.5-injured alveolar epithelial cells. The present study identified that PM2.5 activated the NF- $\kappa$ B signaling pathway and then induced high expression levels of inflammatory factors, such as IL-6, IL-8, IL-1 $\beta$ and TNF- $\alpha$, leading to inflammatory stress in the alveolar epithelial cells. In contrast, OP-D had an anti-inflammatory role via activation of AMPK to inhibit the PM2.5-activated NF- $\kappa B$ pathway. These findings suggested that the anti-inflammatory activity of OP-D may be a valuable strategy for protection against PM2.5- or other cause-induced pulmonary injury progression.

Due to the use of heating systems during the long winter season, air pollution in the city of Changchun is a serious issue during this time period, which markedly increases the concentrations of PM2.5. According to the environmental data released by the Ministry of Ecological Environment of the P.R. China, during the winter of 2017, the concentration of PM2.5 was $60-90 \mu \mathrm{g} / \mathrm{m}^{3}$ in Changchun, which reached 2-3 times that of other seasons in the same area (21), he concentration, 
composition and properties of the particles vary with the region and season. Our previous study found that PM2.5 contained numerous chemical components, including $\mathrm{Al}, \mathrm{Ca}$, $\mathrm{Fe}, \mathrm{K}, \mathrm{Zn}, \mathrm{Mg}$, polycyclic aromatic hydrocarbons, organic carbon and elemental carbon, of which $\mathrm{Al}$ and organic carbon were the most abundant in the PM2.5 samples in Changchun, China, during the winter (22). In the present study, the samples of PM2.5 were collected over five months in winter. Therefore, the present data may be more reflective of what individuals may be exposed to during the winter. Since PM2.5 is composed of extremely complex components, the toxic effects of different batches may reveal different results. Further work will be conducted to observe the anti-inflammatory activity of OP-D on additional samples collected from different cities.

As the first defense barrier between air and lung tissue, the airway epithelium is extremely vulnerable to the stimulation of harmful substances, such as PM2.5 or cigarette smoke. For example, He et al (9) have established that PM2.5 can cause inflammatory effects in MLE-12 cells, which are derived from a female mouse. In addition, multiple studies in male mice have reported that PM2.5 can activate inflammation and lead to lung injury (23-25). Altogether, these findings suggest that sex differences do not affect the outcome of PM2.5 exposure; therefore, the MLE-12 cell line was selected to perform this research.

Previous investigations have demonstrated that PM2.5 can lead to an inflammatory response, which is suggested to be the basic pathogenesis of respiratory disorders of the respiratory system (26) and can damage pulmonary function (27), making the lungs more susceptible to infection. For example, Jeong et al (28) have revealed that the epidermal growth factor receptor/mitogen-activated protein kinase/NF- $\kappa \mathrm{B} / \mathrm{IL}-8$ pathway may be a possible mechanism for PM2.5-induced lung toxicity. In addition, $\mathrm{Li}$ et al (29) observed that PM2.5 may induce inflammatory responses via the Toll-like receptor 4/p38/NF- $\kappa$ B pathway. Notably, activated NF- $\kappa$ B serves a crucial role in PM2.5-induced inflammatory diseases. To date, $\mathrm{NF}-\kappa \mathrm{B}$ is known to consist of five family member protein monomers (p65/RelA, RelB, cRel, p50 and p52) that form homodimers or heterodimers to bind DNA differentially (30). Moreover, p65/RelA is activated and translocated into the nucleus via the formation of different heterodimers with p50 or p52 $(31,32)$. The present study only determined the translocation of $\mathrm{p} 65$, not of the other forms, to investigate the activation of NF- $\mathrm{NB}$. It has previously been reported that PM2.5-induced p65 translocation results in the release of certain inflammatory cytokines (20). After encountering inflammatory irritants, p65, the most important subunit of $\mathrm{NF}-\kappa \mathrm{B}$, undergoes phosphorylation (activation) and translocates to the nucleus, acting as a transcriptional activator to promote the expression of various downstream proinflammatory mediators, including TNF- $\alpha$, IL-6 and IL-1 $\beta$ (8). A previous study revealed that PM2.5 exposure activates the $\mathrm{NF}-\kappa \mathrm{B}$ complex via phosphorylation of nuclear p65 and cytoplasmic IкB kinase- $\alpha$, leading to nuclear p65/p50 DNA binding in human lung epithelial cells in a timeand concentration-dependent manner (33). These findings are consistent with the present results.

In the current study, immunofluorescence staining demonstrated that there was a greater level of NF- $\kappa \mathrm{Bp} 65$ in the nuclei of PM2.5-treated MLE-12 cells, compared with that of the blank control group (Fig. 3). Semi-quantitative analysis via western blotting indicated that the nuclear

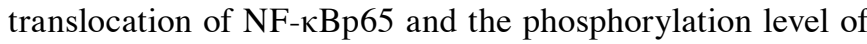
NF- $\kappa$ Bp65 were $~ 3$ (Fig. 4B) and four (Fig. 4A) times greater, compared with the blank control group. In addition, the cytoplasmic NF- $\kappa$ Bp65 was reduced significantly only in the PM2.5-treated MLE-12 cells, compared with that in the blank control group (Fig. 4B). It was also found that PM2.5 stimulation significantly induced the levels of IL-1 $\beta$, IL-6, IL-8 and TNF- $\alpha$ in MLE-12 cells, compared with control cells (Fig. 2). However, the PM2.5-induced inflammatory cytokine response may induce the death of pulmonary epithelial cells, inhibit the junctional gap between these cells to block intercellular communication and impair their function, further leading to alveolar collapse $(34,35)$.

OP-D significantly downregulated the expression of the PM2.5-triggered cytokines in a dose-dependent manner (Fig. 2), indicating that OP-D may have protective effects against PM2.5-induced inflammation. PM2.5-induced nuclear translocation and phosphorylation of $\mathrm{NF}-\kappa \mathrm{Bp} 65$ were partly suppressed by OP-D (Figs. 3 and 4), which suggested that OP-D had a protective effect against PM2.5-induced inflammation via the $\mathrm{NF}-\kappa \mathrm{B}$ signaling pathway.

AMPK is a crucial sensor that regulates the intracellular ATP to AMP ratio in all eukaryotic cells (36). It has been reported that AMPK activation attenuates cigarette smoke-induced inflammatory responses in human lung epithelial cells to protect against the development of emphysema (37). Moreover, activated AMPK inhibits the inflammatory response via the attenuation of $\mathrm{NF}-\kappa \mathrm{B}$ phosphorylation to block the production of proinflammatory cytokines (38). Based on these findings, it was hypothesized that the protective mechanism of OP-D may be partly attributed to activation of AMPK signaling in PM2.5-stimulated MLE-12 cells. Indeed, the present results supported this hypothesis, as it was demonstrated that the activity of OP-D inhibited the release of IL-6, IL- 8, IL- $1 \beta$ and TNF- $\alpha$ via activation of AMPK to block the NF- $\kappa$ B pathway in PM2.5-stimulated MLE-12 cells (Figs. 5 and 6). Furthermore, the AMPK signaling pathway was identified to have a significant protective effect on cellular viability (Fig. 7). However, AMPK activation cannot completely inhibit the activation of $\mathrm{NF}-\kappa \mathrm{B}$ and the production of cytokines, which suggests that other signaling pathways may be involved in the anti-inflammatory effect of OP-D in PM2.5-treated alveolar epithelial cells. Therefore, the specific anti-inflammatory mechanism of OP-D should be investigated in future studies. The present study demonstrated the dose effects of OP-D in the cells; however, whether the doses are equivalent in the in vivo exposure has not been elucidated. Hence, in order to further evaluate the pharmacological effect and value of OP-D, an additional study on the function and dose correlation of OP-D is underway in a mouse model of PM2.5-induced emphysema.

In conclusion, the present study demonstrated that OP-D has anti-inflammatory effects against the PM2.5-induced damage in alveolar epithelial cells. The mechanism of OP-D activity may be via inhibiting the expression of inflammatory cytokines, such as IL-6, IL-8, IL-1 $\beta$ and TNF- $\alpha$, activating phosphorylated AMPK and inhibiting activation of the NF- $\kappa$ B pathway. Therefore, OP-D could potentially be an efficient and 
therapeutic drug for assisting in the treatment of respiratory inflammation caused by PM2.5. The present research also provides theoretical evidence for the transformation of OP-D into clinical applications.

\section{Acknowledgements}

Not applicable.

\section{Funding}

This work was supported by the Project of Science and Technology Agency of Jilin Province (Grant no. 2017J044) and the 59th Chinese postdoctoral program of China (Grant No. 801161020425).

\section{Availability of data and materials}

The data used and/or analyzed during the current study are available from the corresponding author on reasonable request.

\section{Authors' contributions}

HD and YW designed most of the investigation, performed data analysis and wrote the manuscript. YW provided experimental technical assistance, and DL and LS contributed to interpretation of the data and analyses. All authors read and approved the final manuscript.

\section{Ethics approval and consent to participate}

Not applicable.

\section{Patient consent for publication}

Not applicable.

\section{Competing interests}

The authors declare that they have no competing interests.

\section{References}

1. Zheng Q, Liu H, Zhang J and Chen D: The effect of ambient particle matters on hospital admissions for cardiac arrhythmia: A multi-city case-crossover study in China. Environ Health 17 60, 2018.

2. Liao J, Yu H, Xia W, Zhang B, Lu B, Cao Z, Liang S, Hu K, Xu S and $\mathrm{Li} \mathrm{Y}$ : Exposure to ambient fine particulate matter during pregnancy and gestational weight gain. Environ Int 119: 407-412, 2018.

3. Sram RJ, Veleminsky M Jr, Veleminsky M Sr and Stejskalová J: The impact of air pollution to central nervous system in children and adults. Neuro Endocrinol Lett 38: 389-396, 2017.

4. Xing YF, Xu YH, Shi MH and Lian YX: The impact of PM2.5 on the human respiratory system. J Thorac Dis 8: E69-E74, 2016

5. Happo MS, Salonen RO, Hälinen AI, Jalava PI, Pennanen AS, Kosma VM, Sillanpää M, Hillamo R, Brunekreef B, Katsouyanni K, et al: Dose and time dependency of inflammatory responses in the mouse lung to urban air coarse, fine, and ultrafine particles from six European cities. Inhal Toxicol 19: 227-246, 2007.

6. Wang G, Zhao J, Jiang R and Song W: Rat lung response to ozone and fine particulate matter (PM2.5) exposures. Environ Toxicol 30: 343-356, 2015.
7. de F C Lichtenfels AJ, van der Plaat DA, de Jong $\mathrm{K}$, van Diemen CC, Postma DS, Nedeljkovic I, van Duijn CM, Amin N, la Bastide-van Gemert S, de Vries M, et al: Long-term air pollution exposure, genome-wide DNA methylation and lung function in the lifelines cohort study. Environ Health Perspect 126: 027004, 2018.

8. Zhang Y, Wang S, Zhu J, Li C, Zhang T, Liu H, Xu Q, Ye X, Zhou L and Ye L: Effect of atmospheric PM2.5 on expression levels of NF- $\kappa \mathrm{B}$ genes and inflammatory cytokines regulated by $\mathrm{NF}-\kappa \mathrm{B}$ in human macrophage. Inflammation 41: 784-794, 2018.

9. He M, Ichinose T, Yoshida S, Ito T, He C, Yoshida Y, Arashidani K, Takano H, Sun G and Shibamoto T: PM2.5-induced lung inflammation in mice: Differences of inflammatory response in macrophages and type II alveolar cells. J Appl Toxicol 37: 1203-1218, 2017.

10. Peixoto CA, Oliveira WH, Araújo SMDR and Nunes AKS: AMPK activation: Role in the signaling pathways of neuroinflammation and neurodegeneration. Exp Neurol 298: 31-41, 2017.

11. Grahame Hardie D: AMP-activated protein kinase: A key regulator of energy balance with many roles in human disease. J Intern Med 276: 543-559, 2014.

12. Zhang J, Hu Y, Wang D, Qin T, Liu C, Liu X, Sheng X, Chang S, Fan Y, Guo LW and Nguyen TL: The optimization of sulfation modification conditions for ophiopogonpolysaccharide based on antiviral activity. Int J Biol Macromol 51: 657-662, 2012.

13. Liang H, Xing Y, Chen J, Zhang D, Guo S and Wang C: Antimicrobial activities of endophytic fungi isolated from Ophiopogon japonicus (Liliaceae). BMC Complement Altern Med 12: 238, 2012.

14. Kou J, Sun Y, Lin Y, Cheng Z, Zheng W, Yu B and Xu Q: Anti-inflammatory activities of aqueous extract from Radix Ophiopogon japonicus and its two constituents. Biol Pharm Bull 28: 1234-1238, 2005.

15. Chang JM, Shen CC, Huang YL, Chien MY, Ou JC, Shieh BJ and Chen CC: Five new homoisoflavonoids from the tuber of Ophiopogon japonicus. J Nat Prod 65: 1731-1733, 2002.

16. Kou J, Tian Y, Tang Y, Yan J and Yu B: Antithrombotic activities of aqueous extract from Radix Ophiopogon japonicus and its two constituents. Biol Pharm Bull 29: 1267-1270, 2006.

17. Takahama K and Miyata T: Cough-diversity and the peripheral mechanisms of production. Nihon Yakurigaku Zasshi 105: 41-52, 1995 (In Japanese).

18. Qian J, Jiang F, Wang B, Yu Y, Zhang X, Yin Z and Liu C: Ophiopogonin D prevents $\mathrm{H} 2 \mathrm{O} 2$-induced injury in primary human umbilical vein endothelial cells. J Ethnopharmacol 128: 438-445, 2010.

19. Zhang YY, Meng C, Zhang XM, Yuan CH, Wen MD, Chen Z, Dong DC, Gao YH, Liu C and Zhang Z: Ophiopogonin D attenuates doxorubicin-induced autophagic cell death by relieving mitochondrial damage in vitro and in vivo. J Pharmacol Exp Ther 352: 166-174, 2015.

20. Song L, Li D, Li X, Ma L, Bai X, Wen Z, Zhang X, Chen D and Peng L: Exposure to PM2.5 induces aberrant activation of NF- $\mathrm{KB}$ in human airway epithelial cells by downregulating miR-331 expression. Environ Toxicol Pharmacol 50: 192-199, 2017.

21. Monthly report on Urban air quality from the Ministry of ecology and environment of the People's Republic of China. http://www. mee.gov.cn/hjzl/dqhj/cskqzlzky b /index_2.shtml. Accessed August 11, 2020.

22. Song L, Li D, Gu Y, Li X and Peng L: Let-7a modulates particulate matter $(\leq 2.5 \mu \mathrm{m})$-induced oxidative stress and injury in human airway epithelial cells by targeting arginase 2. J Appl Toxicol 36: 1302-1310, 2016.

23. Ogino $\mathrm{K}$, Nagaoka $\mathrm{K}$, Ito $\mathrm{T}$, Takemoto 1 , Okuda $\mathrm{T}$, Nakayama SF, Ogino N, Seki Y, Hamada H, Takashiba S and Fujikura Y: Involvement of PM2.5-bound protein and metals in PM2.5-induced allergic airway inflammation in mice. Inhal Toxicol 30: 498-508, 2018.

24. Ogino K, Nagaoka K, Okuda T, Oka A, Kubo M, Eguchi E and Fujikura Y: PM2.5-induced airway inflammation and hyperresponsiveness in NC/Nga mice. Environ Toxicol 32: 1047-1054. 2017.

25. Yue W, Tong L, Liu X, Weng X, Chen X, Wang D, Dudley SC, Weir EK, Ding W, Lu Z, et al: Short term Pm2.5 exposure caused a robust lung inflammation, vascular remodeling, and exacerbated transition from left ventricular failure to right ventricular hypertrophy. Redox Biol 22: 101161, 2019.

26. Li R, Zhou R and Zhang J: Function of PM2.5 in the pathogenesis of lung cancer and chronic airway inflammatory diseases. Oncol Lett 15: 7506-7514, 2018. 
27. Akther T, Ahmed M, Shohel M, Ferdousi FK and Salam A Particulate matters and gaseous pollutants in indoor environment and association of ultra-fine particulate matters $\left(\mathrm{PM}_{1}\right)$ with lung function. Environ Sci Pollut Res Int 26: 5475-5484, 2019.

28. Jeong SC, Cho Y, Song MK, Lee E and Ryu JC: Epidermal growth factor receptor (EGFR)-MAPK-nuclear factor(NF)-кB-IL8: A possible mechanism of particulate matter(PM) 2.5-induced lung toxicity. Environ Toxicol 32: 1628-1636, 2017.

29. Li R, Zhao L, Tong J, Yan Y and Xu C: Fine particulate matter and sulfur dioxide coexposures induce rat lung pathological injury

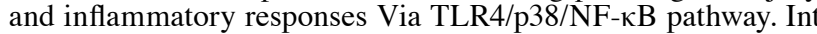
J Toxicol 36: 165-173, 2017

30. Tilborghs S, Corthouts J, Verhoeven Y, Arias D, Rolfo C, Trinh XB and Dam PA: The role of nuclear factor-kappa B signaling in human cervical cancer. Crit Rev Oncol Hematol 120: 141-150, 2017.

31. Leung TH, Hoffmann A and Baltimore D: One nucleotide in a kappaB site can determine cofactor specificity for NF-kappaB dimers. Cell 118: 453-464, 2004.

32. Mukherjee SP, Behar M, Birnbaum HA, Hoffmann A, Wright PE and Ghosh G: Analysis of the RelA: CBP/p300 interaction reveals its involvement in NF- $\kappa \mathrm{B}$-driven transcription. PLoS Biol 11: e1001647, 2013

33. Dagher Z, Garcon G, Billet S, Verdin A, Ledoux F, Courcot D, Aboukais A and Shirali P: Role of nuclear factor-kappa B activation in the adverse effects induced by air pollution particulate matter (PM2.5) in human epithelial lung cells (L132) in culture. J Appl Toxicol 27: 284-290, 2007.
34. Jacquemin B, Lanki T, Yli-Tuomi T, Vallius M, Hoek G, Heinrich J, Timonen K and Pekkanen J: Source category-specific PM2.5 and urinary levels of Clara cell protein CC16. The ULTRA study. Inhal Toxicol 21: 1068-1076, 2009.

35. Longhin E, Holme JA, Gutzkow KB, Arlt VM, Kucab JE, Camatini $\mathrm{M}$ and Gualtieri M: Cell cycle alterations induced by urban PM2.5 in bronchial epithelial cells: Characterization of the process and possible mechanisms involved. Part Fibre Toxicol 10: 63, 2013.

36. Ke R, Xu Q, Li C, Luo L and Huang D: Mechanisms of AMPK in the maintenance of ATP balance during energy metabolism. Cell Biol Int 42: 384-392, 2018.

37. Cheng XY, Li YY, Huang C, Li J and Yao HW: AMP-activated protein kinase reduces inflammatory responses and cellular senescence in pulmonary emphysema. Oncotarget 8: 22513-22523, 2017.

38. Zhang J, Zhang Y, Xiao F, Liu Y, Wang J, Gao H, Rong S, Yao Y, $\mathrm{Li} \mathrm{J}$ and $\mathrm{Xu}$ G: The peroxisome proliferator-activated receptor $\gamma$ agonist pioglitazone prevents NF- $\kappa \mathrm{B}$ activation in cisplatin nephrotoxicity through the reduction of $\mathrm{p} 65$ acetylation via the AMPK-SIRT1/p300 pathway. Biochem Pharmacol 101: 100-111, 2016.

c) (i) $\Theta$ This work is licensed under a Creative Commons Attribution-NonCommercial-NoDerivatives 4.0 International (CC BY-NC-ND 4.0) License. 